\title{
A Study on the Relationship Between the Order of Answering and the Examination Results
}

\author{
Jianjun Cao ${ }^{1, a, *}$, Tianshu Chen ${ }^{1, \mathrm{~b}}$ and Chen Wei ${ }^{1, \mathrm{c}}$
}

\author{
${ }^{1}$ College of Geography and Environmental Science, Northwest Normal University, Lanzhou, Gansu province, China \\ ${ }^{a}$ caojj@nwnu.edu.cn, ${ }^{b}$ cts1435528829@163.com, ${ }^{2} 2018222409 @ n w n u . e d u . c n$ \\ *Corresponding author
}

\begin{abstract}
Examination results are not only affected by intelligence factors, but also affected by non-intelligence factors (such as the order of answering). In order to explore the relationship between the order of answering (including answer questions in order, answer questions in reverse order, from easy to difficult and from difficult to easy) and the examination results, a questionnaire survey was conducted among 465 students (junior high school students, senior high school students and college students, respectively). The results show that: gender and grade affect the selection of order of answering, the order of answering in turn affects the examination results. The students who select answer questions in order had relatively high examination results. The results show that it is necessary to give priority to the answer questions in order for students to achieve higher scores.
\end{abstract}

Keywords: order of answering, examination results, students

\section{答题顺序与考试成绩的关系研究}

\author{
曹建军 ${ }^{1, a}, *$, 陈天舒 ${ }^{1, b}$, 魏晨 ${ }^{1, \mathrm{c}}$
}

\author{
${ }^{1}$ 地理与环境科学学院, 西北师范大学, 兰州, 甘肃省, 中国 \\ ${ }^{a}$ caojj@nwnu.edu.cn, bcts1435528829@163.com,c2018222409@nwnu.edu.cn \\ *通讯作者
}

\begin{abstract}
摘要
考试成绩不仅受智力因素的影响，也受非智力因素（如答题顺序）的干扰。为了探究答题顺序，包括 按题目顺序答题、从易到难、从难到易和逆序与成绩之间的关系，对 465 名在校学生（初中生、高中 生和大学生）进行了问卷调查。结果显示：性别和年级影响答题顺序的选择，而答题顺序的不同反过 来又对成绩产生影响, 其中选择按题目顺序答题的学生成绩相对较高。该结果表明, 为了取得较高的 成绩，学生应优先考虑按题目顺序答题。
\end{abstract}

关键词：答题顺序，考试成绩，学生

\section{1. 引言}

众所周知, 学习成绩与优质教育资源的争取、事业的成功 和个人价值的实现等息息相关。在知识化和竞争激烈的时 代, 学习成绩的重要性更是不言而喻, 尤其在中国的教学 体系中, 考试成绩成了衡量教学质量、教学效率和学生品 行的重要指标之一, 尽管有时学习成绩与能力并不等同。 一般而言, 学习成绩较好的学生, 接受高等教育和获得更 高学位的机会越大。研究表明, 有良好的教育背景的民众 除了高收入回报外, 其身心更健康、寿命更长、犯罪率更 低, 且社会参与度较高 [1]。同时, 他们的自我概念更明 确、乐趣更多、焦虑较少[2]。

从已有的研究看, 影响学生成绩的因素可划分内在因素和 外在因素, 其中内在因素包括智力因素和非智力因素。智 力因素主要反映学生的观察、记忆、思考、想象力、创造 力和其他能力, 而非智力因素通常体现了学生的情感、情
感、意志、气质和性格等[3]。有研究表明, 高年级学生 的成绩往往与这些内在因素有关联, 而低年级学生的学习 一般受外在因素的影响 [4-5], 比如家庭经济状况 [6]、性 别、地理区域、家庭类型、爱好 [7]和学校环境 [8]。另外, 也有研究者指出, 答题顺序这一外在因素也可能影响成 绩。例如, 赵斯明（2013）[9]提出, 在规定时间内答对 最大限度的题量, 是答题顺序安排的基本原则, 合理安排 答题顺序, 有助于拥有良好的答题心理, 从而取得更好的 成绩。刘姝阳（2015）[10]也阐述了答题顺序与成绩存在 直接关系。龙泽勇 (2018) [11]针对中国特色的考试科目 - - 理综, 认为按学科顺序答题比较好, 因为理综是同一 学科内的综合, 而三科的知识体系不同、思维方法不同、 答题的思路也不尽相同。按科目答题, 可以使自己的思路 有个连续性, 从而提高做题的准确性。

然而, 虽然上述研究人员对答题顺序与成绩的关系进行了 定性描述和分析, 但截止目前, 还未见二者关系的实证和 定量研究报道。基于此, 本文以兰州市各校的中学、高中 
和大学生为研究对象, 通过调查和访谈, 对 465 名学生的 答题顺序和考试成绩等情况进行分析, 以为探究考试成绩 和答题顺序的关系和提高教学管理质量提供思路和方法。

\section{2. 研究方法}

\section{1. 调查方法}

采用调查问卷的方法, 以兰州市不同学校的初中生、高中 生、大学生为调查对象, 对其性别、教育背景、答题顺序 （包括 A、按题目顺序; B、从难到易；C 从易到难；D、 逆序。) 和英语成绩进行了调查, 因为中学阶段和大学阶 段英语考察的内容和形式基本一致。本次收回有效问卷 465 份, 其中初中生 110 份, 高中生 120 份, 大学生 235 份。

\section{2. 分析方法}

应用 SPSS22.0 统计分析软件, 样本均数间的比较采用 $\mathrm{t}$ 检验, 构成比差异用列联表 $\mathrm{X} 2$ 检验, 显著性水平为 $\mathrm{P}<$ 0.05 。

\section{3. 结果}

\section{1. 不同答题顺序的平均分及成绩分布}
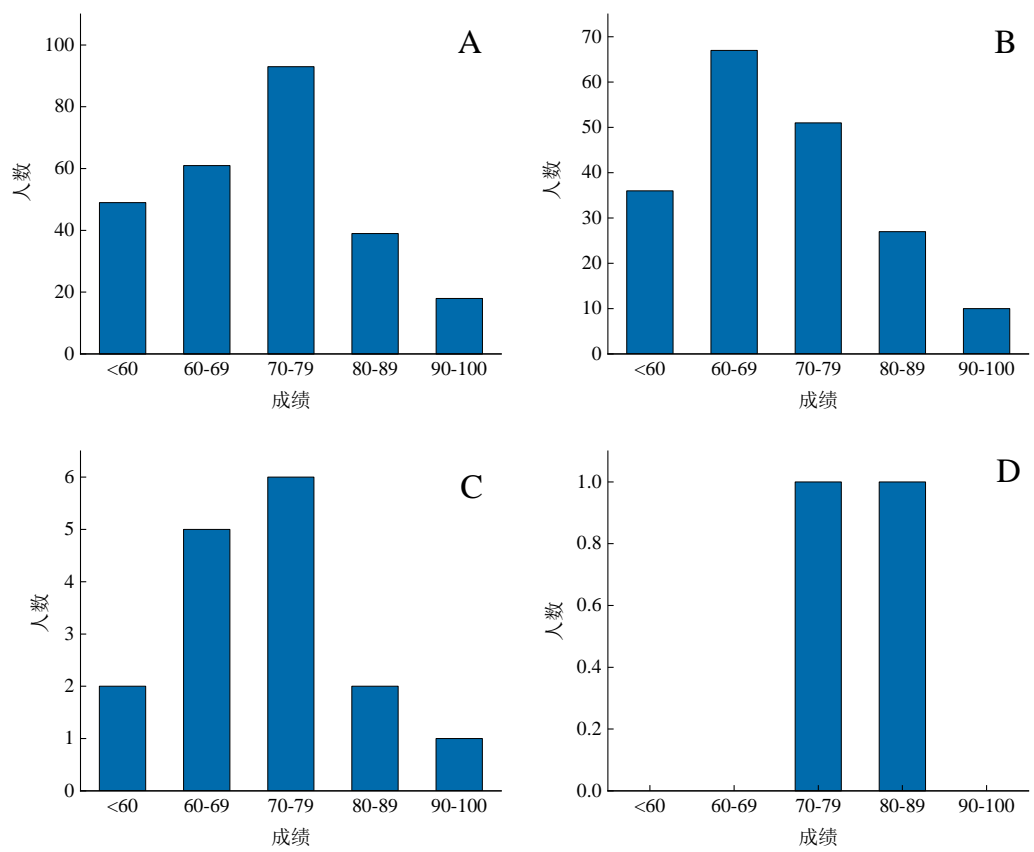

图 1 不同答题顺序的考试成绩分布

A 表示按题目顺序答题; B 表示从易到难；C 表示从难到易； D 表示逆序
不同的答题顺序对成绩有一定的影响, 差异有统计学意 义。使用独立样本 $\mathrm{t}$ 检验进行统计检验, 以减少抽样误差 (表 1)。

由表 2 可知, 选项 $\mathrm{A}$ 的平均分显著高于选项 C 和选项 B, 表明选择 $\mathrm{A}$ 选项能让学生取得更高的考试成绩, 而选择 选项 $\mathrm{B}$ 和选项 $\mathrm{C}$ 的学生考试成绩无显著差异。

表 1 不同答题顺序对成绩的影响 (独立样本 $\mathrm{t}$ 检验结果)

\begin{tabular}{ccc}
\hline 选项 & Sig & Sig (双侧) \\
\hline $\mathrm{A}$ 与 $\mathrm{B}$ & $<0.05$ & 0.004 \\
$\mathrm{~A}$ 与 C & $<0.05$ & 0.027 \\
$\mathrm{~B}$ 与 $\mathrm{C}$ & $<0.05$ & 0.022 \\
\hline
\end{tabular}

表 2 不同答题顺序的样本数和平均分

\begin{tabular}{ccc}
\hline 选项 & 样本数 $(\mathrm{N})$ & 平均分 \\
\hline A & 257 & $72.78 \pm 14.25$ \\
B & 190 & $69.23 \pm 12.15$ \\
C & 16 & $69.93 \pm 9.82$ \\
\hline & 注: A 表示按题目顺序答题; B 表示
\end{tabular}

从易到难; $\mathrm{C}$ 表示从难到易

选择 $\mathrm{A}$ 选项的人数在 70-79 分段的人数最多，有 93 人; 选择 B 选项的人数在 60-69 分段的人数最多, 有 67 人; 选择 $\mathrm{C}$ 选项的人数在 70-79 分段的人数最多, 有 6 人。选 择 $\mathrm{A}$ 选项、 $\mathrm{C}$ 选项的学生考试成绩主要集中于中上水平 （70-79），选择 B 选项的学生成绩主要集中于中下水平 (60-69)（图 1）。 
总人数的 $71.1 \%$; 选择 $\mathrm{C}$ 选项的女生有 5 名, 占选项 $\mathrm{C}$

\section{2. 性别、年级影响答题顺序}

465 名受试者中, 男生 234 名, 女生 231 名。选择 A 选项 的女生有 170 名, 占选项 A 总人数的 $66.1 \%$, 男生有 87 名, 占选项 A 总人数的 $33.9 \%$; 选择 $\mathrm{B}$ 选项的女生 55 名, 占选项 B 总人数的 $28.9 \%$, 男生有 135 名, 占选项 B
总人数的 $31.3 \%$, 男生有 11 名, 占选项 C 总人数的 $68.7 \%$; 选择 D 选项的男生 1 名, 女生 1 名, 各占选项 D 总人数 的 $50.0 \%$ 。女生选择 A 选项所占的比例最高, 而男生选 择 $\mathrm{B}$ 选项的比例最高, 且女生考试成绩的平均分高于男 生（表 3、表 4）。

表 3 答题顺序和性别之间的关系

\begin{tabular}{ccccccc}
\hline & $\mathrm{A}$ & $\mathrm{B}$ & $\mathrm{C}$ & $\mathrm{D}$ & $\chi^{2}$ & $\mathrm{P}$ 值 \\
\hline 男 & 87 & 135 & 11 & 1 & 62.72 & $<0.05$ \\
女 & 170 & 55 & 5 & 1 & & \\
合计 & 257 & 190 & 16 & 2 & & \\
\hline
\end{tabular}

A 表示按题目顺序答题; B 表示从易到难; C 表示从难到易; D 表示逆序

表 4 男女生考试成绩的平均分

\begin{tabular}{cc}
\hline 分组 & 平均分 \\
\hline 男 & 68.25 \\
女 & 70.83 \\
\hline
\end{tabular}

不同年级的学生在答题顺序的选择上具有一定差异, 差异 具有统计学意义 $(\mathrm{P}<0.05)$ 。就年级来说, 选择 $\mathrm{A}$ 选项的 学生中, 高中生人数所占比重最大, 初中生和大学生次之; 选择 $\mathrm{B}$ 选项的学生中, 大学生人数所占比重最大, 初中 生和高中生次之; 选择 $\mathrm{C}$ 选项的学生中, 大学生人数所 占比重最大（表 5、表 6）。

表 5 不同年级的答题顺序选择的差异性

\begin{tabular}{ccc}
\hline 分组 & $\chi 2$ & $\mathrm{P}$ 值 \\
\hline 初中与高中 & 41.27 & $<0.05$ \\
初中与大学 & 22.43 & $<0.05$ \\
高中与大学 & 25.31 & $<0.05$ \\
\hline
\end{tabular}

表 6 各选项间不同年级所占人数比重比较

\begin{tabular}{cc}
\hline 选项 & 各年级人数所占比重比较 \\
\hline A & 高中 $>$ 初中 $>$ 大学 \\
B & 大学 $>$ 初中 $>$ 高中 \\
C & 大学 $>$ 高中 \\
\hline
\end{tabular}

示从易到难; $\mathrm{C}$ 表示从难到易

\section{4. 讨论}

\section{1. 答题顺序对平均分及成绩分布的影响}

研究表明答题顺序影响学生的考试成绩[12-13]。本研究 中, 选择 $\mathrm{A}$ 选项的学生考试成绩的平均分高于 B、C 选
项的考试成绩, 是因为这些学生节奏把握较好, 掌握知识 比较扎实, 能有效减少漏题等低级错误出现的概率, 心态 较为平缓, 能确保正常水平的发挥。选择 $\mathrm{B}$ 选项和 $\mathrm{C}$ 选 项的学生考试成绩的平均分无显著差异, 表明 B 选项和 C 选项对学生考试成绩的影响相当。与学生个人喜好, 所处 年级阶段有关, 访谈发现有部分同学偏向于选择 C, 但由 于一开始没有解答出难题导致考试心态失衡, 继而影响到 后续的做题节奏和心情，从而发挥失常、考试成绩较低; 此外, 访谈中发现选择 $\mathrm{B}$ 选项的初中生因平时掌握基础 知识薄弱, 在考试中不会的题目较多, 只能选择相对简单 的做, 而高中、大学阶段的同学选择 $\mathrm{B}$ 选项是因为题量 大、题目难度较高。然而, 就考试成绩分布来看, 选择 $\mathrm{A}$ 选项、 $\mathrm{C}$ 选项的学生考试成绩处于中上水平, 这说明 $\mathrm{A}$ 选项（原因在前已说明）、C 选项有利于取得更高的考试 成绩, 是因为选择 $\mathrm{C}$ 的学生大多数是能力较强、喜欢先 做有挑战的事情的同学 [14], 也有一部分同学是认为自己 在考试最开始精力最好、记忆最清楚, 最有可能答出难题 或者从难题中得到一部分分数。

\section{2. 性别、年级对考试成绩的影响}

性别差异对答题顺序的选择产生影响最终影响学生的考 试成绩[15]。女生选择 A 选项的比例最高, 且女生考试成 绩的平均分高于男生。这与目前国内的研究结果相似, 但 与国外的研究有一定的区别, 国外的一些研究发现女生的 考试成绩相对而言低于男生, 这与我们国家的文化氛围及 考试对学生个人的重要程度有关[15]。本研究中, 女生的 考试成绩整体优于男生，与兰君。(2016) [16]和 Aurah. (2017) [17]的研究结果一致, 这与女生的性格等个人特质 有关, 女生做题一般比较细心周道, 大多数女同学都有正 确的学习动机、稳定的学习情绪、持久的学习行为和顽强 的学习意志 [18-20]。另外, 男女同学在获取知识途径的认 识上有所不同, 女生更加重视理论知识的学习, 更适应卷 面考试[21-22]。男生通常粗心大意, 没有顽强的学习意志, 且男生更注重实践, 理论知识薄弱, 使得其考试成绩的平 均分低于女生[23]。这也反映出女生尤其是当代女大学生 意识到自身生存与发展的过程中可能遇到比男生更多的 困难，诸如就业立业、家庭负担等，通过提高考试成绩， 为自己争取到了更多的接受教育的机会, 在以后激烈的社 会竞争中立于不败之地。 
Procrastination Scale[J]. 2019, 42-45.

[4] 马红彪, 王正华.初中学生学习成绩影响因素研究 [J]. 中国校医，2002，2(16): 112-114.

[5] 管益杰.关于家庭因素与儿童问题行为关系的初步探 讨 $[J]$.山东师范大学学报: 人文社会科学版(6 期), 1998, 71-74.

[6] Carnoy, Rothstein."International Tests Show Achievement Gaps in All Countries", Economic Policy Institute, 2013.

[7] 郭颖, 裴冬梅.大学生课堂出勤,随堂测试成绩与期末 考试成绩的关系研究 [J].卫生职业教育, 2019， 14(37): 41-42.

[8] 李克俊, 王正华.非主观因素对大学生学习成绩影响的 方差分析[J]. 四川教育学院学报, 2008.

[9] 赵斯明, 孟庆忠. 2013 年高考新增词汇分析与备考 [J]. 新高考(英语进阶)，2013，（3)：8-11.

[10] 刘姝阳. 班主任效应在化学学科教学中的建立及应 用[D]. 黑龙江：哈尔滨师范大学，2015.

[11] 龙泽勇.浅谈高考理科综合答题技巧. 中国校外教育, 2018，639(19): 40-45.

[12] 王亚联.对大学生阅读理解答题顺序的初步探讨. 文 学界(理论版)(01)，2011，168-169.

[13] 向奎.高考理科综合考试中的时间安排和答题顺序之 我见. 网络导报.在线教育(20)，2012，43-44.

[14] Willms, Douglas J. The balance thesis: contextual effects of ability on pupils' o - grade examination results[J]. Oxford Review of Education, 1985, 11(1): 33-41.

[15] 蔡正兵.基于思想政治考试成绩性别差异的教学与评 价研究. 江苏省: 南京师范大学, 2015 .

[16] 兰君.大学生中男女生学习成绩差异比较研究 [J]. 2016, 37(1).

[17] Aurah C. Investigating the relationship between science self-efficacy beliefs, gender, and academic achievement, among high school students in kenya. Journal of Education and Practice, 2017, 8.

[18] 周艳. 大学生英语成绩的性别差异探究 [J]. 安徽工业 大学学报(社会科学版), 2005, (3)。

[19] 李瑾.影响大学生学习成绩因素分析 [J].天津成人高 等学校联合学报, 2005, (3).

[20] 梅少粉, 代亚男.大学生课堂学习有效性的影响因素 探析[J].新教育时代: 教师版，2016，(44):73.

[21] Veerasamy AK, D’Souza D, Lindén R. The Impact of Prior Programming Knowledge on Lecture Attendance and Final Exam[J]. Journal of Educational Computing Research, 2018, 56(2):226-253.

[22] 顾顺奎.试说男女生学习成绩差异的主因 [J].浙江经 济高等专科学校学报, 1998, (2).

[23] Acar Ö, Türkmen L, Bilgin A. Examination of gender differences on cognitive and motivational factors that influence 8th graders' science achievement in turkey. Eurasia Journal of Mathematics Science \& Technology Education, 2015, 11, 1027-1040.

[24] Malhotra T. Exam Anxiety Among Senior Secondary School students. Scholarly research journal for interdisciplinary studies Vaish College of Education, Rohtak, Haryana. 2015.

[25] 李红春, 孔峰. 2018 年全国高考卷 I 试题评析及教学 建议. 中小学数学(高中版)(6), 2018。 
[26] 骆君. 关于初中物理试卷命题的探讨. 南北桥(9), 2016.

[27] 王莉, 任亮宝, 赵爱. 大学期末考试试卷定性分析研 究. 河西学院学报(01), 2011, 130-134. 